\section{Model for Predicting Temporomandibular Dysfunction: Use of Classification Tree Analysis}

\author{
Jorge P. Waked ${ }^{1}$, Mariana P. L. de A. M. Canuto $^{2}$, Maria Cecilia S. N. \\ Gueiros $^{2}$, João Marcílio C. N. L. Aroucha ${ }^{2}$, Cleysiane G. Farias ${ }^{2 \mathbb{D}}$, Arnaldo \\ de F. Caldas $\mathrm{Jr}^{2}{ }^{\mathbb{D}}$
}

${ }^{1}$ Center for Rural Health and Technology, Academic Unit of Biological Sciences, UFCG Universidade Federal de Campina Grande, Patos, PB, Brazil ${ }^{2}$ Health Science Center, Department of Clinical and Preventive Dentistry, UFPE - Universidade Federal de Pernambuco, Recife, PE, Brazil

Correspondence: Arnaldo de F. Caldas Jr, Avenida Professor Moraes Rego, 1235, 50670-901 Recife, PE, Brasil. Tel: +55-81-99971-3652. e-mail: caldasjr@alldeia.com.br
The aim of this study was to construct a predictive model that uses classification tree statistical analysis to predict the occurrence of temporomandibular disorder, by dividing the sample into groups of high and low risk for the development of the disease. The use of predictive statistical approaches that facilitate the process of recognizing and/or predicting the occurrence of temporomandibular disorder is of interest to the scientific community, for the purpose of providing patients with more adequate solutions in each case. This was a cross-sectional analytical population-based study that involved a sample of 776 individuals who had sought medical or dental attendance at the Family Health Units in Recife, PE, Brazil. The sample was submitted to anamnesis using the instrument Research Diagnostic Criteria for Temporomandibular Disorders. The data were inserted into the software Statistical Package for the Social Sciences 20.0 and analyzed by the Pearson Chi-square test for bivariate analysis, and by the classification tree method for the multivariate analysis. Temporomandibular disorder could be predicted by orofacial pain, age and depression. The high-risk group was composed of individuals with orofacial pain, those between the ages of 25 and 59 years and those who presented depression. The low risk group was composed of individuals without orofacial pain. The authors were able to conclude that the best predictor for temporomandibular disorder was orofacial pain, and that the predictive model proposed by the classification tree could be applied as a tool for simplifying decision making relative to the occurrence of temporomandibular disorder.
Key Words: temporomandibular joint dysfunction syndrome, analysis of decisions, decision trees, decision support techniques.

\section{Introduction}

Specifically in the case of temporomadibular disorder (TMD), which has a multifactorial etiology, the presentation of muscle and joint diagnoses, multiple treatment alternatives and relations with various co-morbidities may be observed (1). Epidemiological researches have conducted in-depth investigations into the variables related to TMD, and have demonstrated that physical, socioeconomic, demographic and psychosocial factors appeared to have great influence on its prevalence. These factors may be represented by gender (2-3); age (4), educational level; economic class; family income (5), presence of pain, and depression $(6,7)$.

The female gender has been pointed out as that with the highest prevalence in the majority of studies that involved TMD, and this has also been consider a predisposing factor for occurrence of the dysfunction (2-3,8). Biological, sociocultural and psychosocial circumstances have been pointed out as possible explanations. In the literature, it is also common to find that relative to age, the age-range distribution of patients with TMD is characterized by a Gauss curve. Studies have noted that there is a peak prevalence between 35 and 45 years of age, and a reduction in younger persons and at more advanced ages $(2-3,8)$.

Inaccessibility to education, income, leisure, food and health service are circumstances that may have a repercussion on factors of stress in individuals, and contribute to the appearance, maintenance and progression of changes such as myofascial pain and joint problems of temporomandibular dysfunctions $(5,9)$.

In addition to the socioeconomic variables, the condition of pain involved in temporomandibular dysfunctions is of extreme relevance, because it interferes negatively in the day to day life of patients. When pain assumes a chronic nature, there is greater functional compromise and a rise in the levels of depression and anxiety (9). Pain has been pointed out as being the main cause of any association between TMD and psychosocial symptoms (10).

Given the potentially complex circumstances, individuals with TMD pose a challenge to dentists, because they frequently present with a disturbing combination of clinical, psychological and psychosocial manifestations. For these reasons, from the epidemiological point of view, multivariate approaches to factors associated with TMD are necessary. Instead of favoring predictive models, there has been a predominance of traditional statistical modeling by 
means of multivariate analyses, such as logistic regressions and hierarchical models (5). While explanatory models are retrospective and capable of pointing out the main risk factors for TMD, predictive modeling is prospective and adequate for providing insight into which variables may be predictive for TMD (11).

The use of structured statistical approaches is in the interest of the scientific community, since they facilitate the process of recognition and/or prediction of the occurrence of TMD, with the purpose of providing patients with more adequate solutions for each case. In this context, the use of the decision tree fits in as method of decisive analysis that would facilitate this task $(11,12)$.

Decision trees are considered graphic tools that provide users with a clear view of the variables and their multiple alternatives of associations and outcomes $(11,13)$. They are used in the field of health primordially to resolve three types of clinical difficulties: diagnosis, choice of therapy, and prognosis of events in health. At the top of the structure, there is the root, from which all the observations of the sample are ramified, and the knots produced in sequence represent the subdivisions of data into groups. The variable most strongly associated with the outcome variable is used as the test in each ramification (13). Thus, their purpose is to segment populations and define sub-groups that share similar characteristics, whether they are of risk or protection for events in health, and thus, they fulfill the principle of equity in the supply of promotion, prevention and care in health (14). Since the classification tree is an option to predict health and disease events, it is capable to establish a final model of prediction even in cross-sectionals studies.

In Dentistry, experiences have been reported, particularly relative to clinical situations in the fields of cariology (15) and epidemiology (13). However, in the field of orofacial pain and temporomandibular dysfunction, searching at PubMed from 2000 to 2019, no studies were found using the classification tree for predicting TMD by means of segmentation between groups of high and low risk for its development.

In the case of TMD in any patient, there is the possibility of various overlapping diagnoses or conditions with the potential to mimic orofacial pain. The differentiation between multiple conditions makes the diagnostic process complex, and this frequently leads to it being approached with a certain degree of uncertainty. Moreover, therapeutic decisions that emanate from the diagnostic process have their own predictive uncertainties. There are no possibilities of eliminating uncertainties, however, by recognizing them, strategies may be developed to control and minimize their negative effects (16).

Therefore, the aim of this study was to propose a model for predicting TMD, by using the classification tree and identifying groups at high and low risk for development of TMD in the city of Recife, PE, Brazil. Our study hypothesis was the decision tree is capable to predict the main risk factors associated with TMD.

\section{Material and Methods}

In this study, a total of seven hundred and seventy-six patients participated. They were included in the sample in accordance with the following eligibility criteria: age equal to or over 15 years; must be registered with the Family Health Unit - FHU - of the Brazilian national health system "Sistema Único de Saúde - SUS", must have been drawn and agreed participate in the study. Furthermore, the participants had to be capable of speaking, reading and/or understanding the Portuguese language; must not present acute pain of odontogenic origin and otorhynolaryngological origin; must not have history of rheumatological pathologies or atypical pains. Excluded from the study were those with neurological disturbances or history of tumors in the region of the head and neck, and individuals under the age of 18 years who were not accompanied by a parent or guardian. All health information was extracted from the patient's records.

The sample size calculation was based on the conclusion of a previous pilot study (5) that indicated a prevalence of $42 \%$ for TMD and was used an error of 5\% and a confidence interval of $95 \%$. All those values were used to calculate the sample size. Multi-stage sampling was used to determine the sample size and obtain a representative sample of the entire city. First, systematic sampling was employed to define the neighborhoods in the health districts that would take part in the study. Systematic sampling was then performed to select the Primary Health Units, from which 776 volunteers at their respective health units were randomly selected.

\section{Procedure and Instrument}

This was a population-based, analytical cross-sectional study, in which the patients were approached on the premises of the 51 family health units invited to participate in the research. The investigation was conducted by 4 dentists who were previously trained and calibrated in the application of the Research Diagnostic Criteria for TMD (RDC/TMD), obtaining an intra- and inter-examiner kappa index of 1.0 and 0.8 ; respectively, for the diagnostic variable in TMD. All the individuals selected were submitted to anamnesis and intra- and extraoral clinical exam, in a private room, while seated in dental chairs. A questionnaire containing socioeconomic and demographic information and Axes I and II of the RDC/TMD - Research Diagnostic Criteria for TMD were applied (17).

The RDC/TMD has two axes of evaluation, I and II that 
evaluate the presence of TMD and the psychosocial and behavioral information, respectively, about the individuals. Axis I contains 10 questions that are answered by means of a clinical exam, and after this has been performed, leads to the diagnosis of two types of TMD. Whereas, Axis II, composed of 31 questions, evaluates the biobehavioral context of the individual, and present information relative to the patient's psychological state. For the economic classification, the Brazilian Economic Classification Criteria (BECC), designed by the Brazilian Association of Research Companies, was used.

\section{Variables Investigated}

The dependent variable was TMD, obtained by means of Axis I of the RDC/TMD. TMD presents the following diagnoses: Myofascial pain with or without limitation of mouth opening (Group 1-G1); disc displacement with and without reduction and with or without limitation of opening (Group 2-G2), and arthralgia, osteoarthritis and osteoarthrosis (Group 3-G3). Individuals diagnosed with any of these conditions were considered affected by TMD.

The independent variables were of a socioeconomic; demographic; psychosocial and clinical nature, treated in a dichotomous manner. The sociodemographic characteristics were age; gender; educational level; self-reported skin color; marital status; economic class and family income per capita. Age was categorized, based on adaptations to the World Health Organization (WHO) criteria for oral exams corresponding to the following age-ranges: 15 to $18 ; 19$ to $24 ; 25$ to $44 ; 45$ to 59 , and 60 years or older (18).

For the psychosocial variable depression, question 20 of Axis II of the RDC/TMD was used, composed of a psychometric evaluation scale made up of 32 items. Evaluation is made by the mean value of the sum of scores of each response and in comparison with a score pre-established by the RDC/TMD, classified into absent, moderate or severe.

The clinical variable orofacial pain resulted from categorization of the four main questions of Axis I of the $\mathrm{RDC} / \mathrm{TMD}$ that involved pain, namely questions $1,8,9$ and 10. Question 1 refers to pain in the face self-reported by the individual. Questions 8,9 and 10 were related to pain present at the clinical exam performed by the examiners, who performed intra and extra-oral palpation in different areas of the face. Thus, the reference to pain in any of the questions, or in the set of questions characterized the variable orofacial pain.

\section{Statistical Analysis}

The database was created in IBM SPSS version 20.0 for Windows software (Statistical Package for the Social Sciences). Descriptive analysis of TMD was performed according to the independent variables investigated, and the association of these by means of the Chi-square test. The odds ratio was calculated to measure the degree of significance of the independent variables on the dependent variable TMD.

The multivariate classification tree model was induced by means of the algorithm Chi-square Automatic Interaction Detector (CHAID) that is based on the Chi-square test to identify which variables are of most importance to the classification. The independent variable that presented the greatest interaction with the dependent variable was elected in each step of the induction of CT. Based on the tree, the set of classification rules could also be obtained; that is, the textual representation of the tree that is extracted when it runs in the direction from the root knot to the leaf knots.

As basic parameters for generating the model of prediction, the level of significance was determined for the division of the knots and fusion of the categories of 0.10 . Pearson's statistics was used as the method for obtaining the Chi-square value; maximum number of iterations and minimum change in the expected frequencies of the cells of 0.001 for estimating the model. The significance values for the methods, parameters of division and fusion were corrected by the Bonferroni method.

The limits of growth were defined based on the maximum depth of the tree and on the minimum number of cases. The maximum number of three levels was adopted for the algorithms, and the minimum number of cases was determined as 100 for the root knot and 50 for the derived knots. These parameters were established to try and obtain the highest number of interactions among the variables.

So that the trees of decision will be used in a generalized form, in larger populations, these must be submitted to an evaluation of the quality of the structure. This process is called validation and may be performed in a crossed manner or by sample division. In this study cross validation was adopted, with the use of ten sub-groups for comparison.

In this validation the sample is divided into various sub-samples or folds. Consequently, various models of trees are generated by always successively excluding a sub-group of data. For each tree, the risk of classification error is calculated, by applying the tree to the subsample excluded in generating it. As the final result, one single tree is obtained, whose risk estimate is calculated by the mean value of risks for all the trees, indicating the power of prediction of the tree so that it may be used in a generalized manner.

\section{Results}

Table 1 describes temporomandibular dysfunction according to the following variables: socioeconomic; 
demographic; presence of orofacial pain and depression. The prevalence of TMD 35.4\% $(n=275)$. There was statistically significant difference in this prevalence between the variables sex; age; depression, and presence of orofacial pain. A higher level of occurrence of TMD was observed for the female sex, with greater expressiveness in the age ranges from 45 to 59 years associated with depression; and
80.4\% of the patients with TMD presented orofacial pain. With reference to the other, marital status; self-reported skin color; educational level; income, and BECC were not associated with the outcome.

Figure 1 presents the results of the classification tree generated by the CHAID algorithm. The tree was composed of 7 knots, 4 being terminal knots, and by three levels of

Table 1. Distribution of TMD according to sociodemographic characteristics, presence of orofacial pain and depression

\begin{tabular}{|c|c|c|c|c|c|c|c|c|c|c|}
\hline \multirow{2}{*}{ Variables } & \multicolumn{2}{|c|}{ TMD } & \multicolumn{2}{|c|}{ No TMD } & \multicolumn{2}{|c|}{ Total } & \multirow{2}{*}{$\begin{array}{c}\mathrm{p} \\
\text { value }^{*}\end{array}$} & \multirow{2}{*}{ OR } & \multirow{2}{*}{\multicolumn{2}{|c|}{ CI95\% }} \\
\hline & $\mathrm{n}$ & $\%$ & $\mathrm{n}$ & $\%$ & $\mathrm{n}$ & $\%$ & & & & \\
\hline \multicolumn{11}{|l|}{ Sex } \\
\hline Male & 32 & 26.6 & 88 & 73.3 & 120 & 100 & 0.037 & 1.62 & 1.05 & 2.50 \\
\hline Female & 243 & 37 & 413 & 63 & 656 & 100 & & & & \\
\hline \multicolumn{11}{|l|}{ Age } \\
\hline 5 to 18 years & 10 & 25.6 & 29 & 74.3 & 39 & 100 & 0.032 & - & - & - \\
\hline 19 to 24 years & 29 & 33.3 & 58 & 66.6 & 87 & 100 & & & & \\
\hline 26 to 44 years & 132 & 36.4 & 230 & 63.5 & 362 & 100 & & & & \\
\hline 45 to 59 years & 87 & 41 & 125 & 59 & 212 & 100 & & & & \\
\hline 60 or older & 17 & 22.3 & 59 & 77.6 & 76 & 100 & & & & \\
\hline \multicolumn{11}{|l|}{ Marital Status } \\
\hline Single & 47 & 32.6 & 97 & 67.4 & 144 & 100 & 0.495 & 0.86 & 0.58 & 1.26 \\
\hline Others & 228 & 36 & 404 & 64 & 632 & 100 & & & & \\
\hline \multicolumn{11}{|l|}{ Color } \\
\hline Black & 70 & 41 & 101 & 59 & 171 & 100 & 0.107 & 0.74 & 0.52 & 1.05 \\
\hline Others & 205 & 33.9 & 400 & 66.1 & 605 & 100 & & & & \\
\hline \multicolumn{11}{|l|}{ Educational level } \\
\hline Complete up to Primary School & 144 & 34.5 & 273 & 65.5 & 417 & 100 & 0.622 & 1.09 & 0.81 & 1.46 \\
\hline As from High School & 131 & 36.5 & 228 & 63.5 & 359 & 100 & & & & \\
\hline \multicolumn{11}{|l|}{ Income } \\
\hline Up to $\mathrm{R} \$ 3,200.00^{* *}$ & 263 & 35.2 & 484 & 64.8 & 747 & 100 & 0.592 & 1.35 & 0.6 & 2.98 \\
\hline Over $\mathrm{R} \$ 3,200.00^{* *}$ & 11 & 42.3 & 15 & 57.7 & 26 & 100 & & & & \\
\hline Not informed & 1 & 33.3 & 2 & 66.7 & 3 & 100 & & & & \\
\hline \multicolumn{11}{|l|}{$\mathrm{BECC} * * *$} \\
\hline $\mathrm{A} / \mathrm{B}$ & 34 & 45.4 & 41 & 54.6 & 75 & 100 & 0.094 & - & - & - \\
\hline $\mathrm{C}$ & 164 & 33 & 332 & 77 & 496 & 100 & & & & \\
\hline $\mathrm{D} / \mathrm{E}$ & 76 & 59.4 & 128 & 40.6 & 204 & 100 & & & & \\
\hline \multicolumn{11}{|l|}{ Depression } \\
\hline Absence & 74 & 26.3 & 207 & 73.6 & 281 & 100 & 0.000 & 1.91 & 1.39 & 2.63 \\
\hline Presence & 201 & 40.6 & 294 & 59.4 & 495 & 100 & & & & \\
\hline \multicolumn{11}{|l|}{ Orofacial Pain } \\
\hline No & 54 & 17.6 & 252 & 82.3 & 306 & 100 & 0.000 & 4.14 & 2.93 & 5.85 \\
\hline Yes & 221 & 47 & 249 & 52.9 & 470 & 100 & & & & \\
\hline
\end{tabular}

${ }^{*}$ Chi-square test and the Pearson correlation with correction for continuity; **Brazilian Reais; ${ }^{* * * *}$ BECC: Brazilian Economic Classification Criteria. 
depth. TMD could be predicted by orofacial pain; age, and depression. It is important to note the root knot with the total number of participants in the sample, of whom 35.4\% presented TMD. The variable that most discriminated the root knot was the presence of orofacial pain, forming two distinct groups for predicting TMD. Generally speaking, TMD was more prevalent in individuals with orofacial pain, with age from 25 to 59 years and who presented depression,

TMD

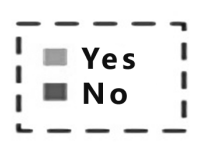

\begin{tabular}{|lrr|}
\hline \multicolumn{4}{|c|}{ Node 0} \\
Category & $\%$ & $n$ \\
\hline Yes & 35.4 & 275 \\
No & 64.6 & 501 \\
\hline Total & 100.0 & 776 \\
\hline \multicolumn{4}{|c|}{} & & \\
\hline & PAIN &
\end{tabular}

Adj. P-value $=0.000$. Chi-square $=69$.

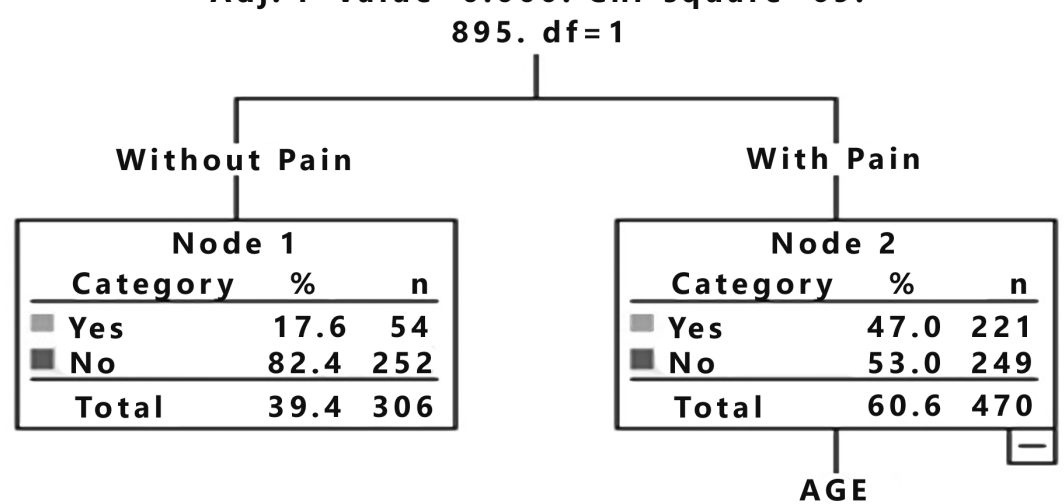

Adj. P-value=0.005. Chi-square $=12$.

$$
\text { 754. } d f=1
$$

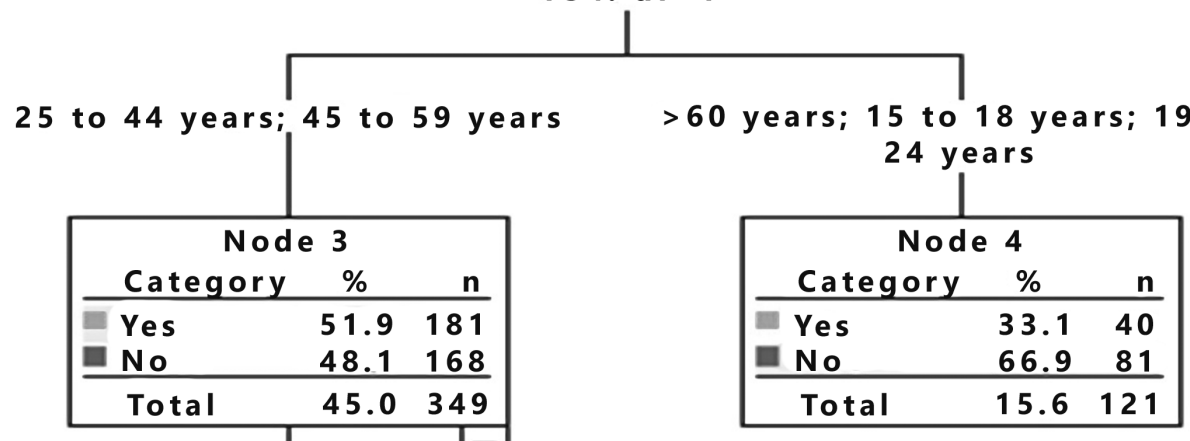

DEPRESSION

Adj. $P-v a l u e=0.021$. Chi - square $=5$.

328. $d f=1$

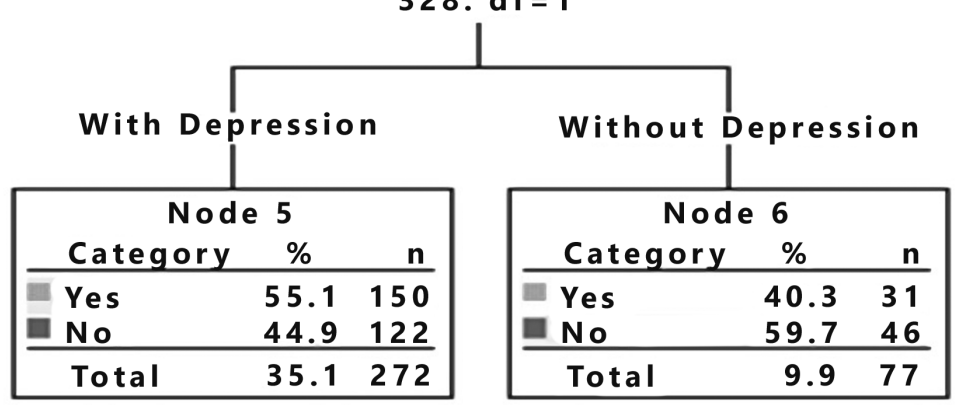

Figure 1. Model for predicting TMD classification tree CHAID algorithm. 
thus composing the group at high risk for the development of TMD in the studied sample. The low risk group were the individuals without orofacial pain.

The classification rules resulting from the textual representation of the tree were the following: if the individual did not present orofacial pain, then the probability of TMD occurring would be $17.6 \%$ and this did not depend on other variables (first terminal knot). If the individual had orofacial pain, then the prediction of TMD was $47 \%$, and also depended on age. If the age were from 15 to 24 years or from 60 or older, the probability was $33.1 \%$ and did not depend on another characteristic. But if the age ranged from 25 to 59 years, the probability of having TMD would be $51.9 \%$ and would also depend on depression. If the subject were not depressive, the probability of TMD would be $40.3 \%$, however if the individual had depression, the probability of TMD would be $55.1 \%$. The global power of prediction of the tree was 0.682 ; that is the tree would predict correctly in $68.2 \%$ of the times.

\section{Discussion}

The originality of the present study is owing to the proposal of a predictive model that used the classification tree to optimize the prediction of TMD, with a sample divided into groups of high and low risk for the development of TMD. Being capable of predicting the probability of an occurrence of a disease has been a challenge that has instigated medical researchers. Nevertheless, the approaches that are capable of predicting diseases, based on statistical and data mining methods, comprise an area that has, as yet, hardly been explored in biomedical researches $(11,12)$.

In practice, predictive analysis applies statistical algorithms to historical data to identify the probability of occurrence of a certain condition. Therefore, the model is constructed to predict new observations. When applied to public health, the analysis is important because it helps with perception of the possibility of a certain individual fitting into a pathological condition that is difficult to diagnose, such as TMD. These models prove their reliability because they are constructed from analysis of explanatory variables, and among these, they verify those that are also predictors of the disease $(6,11)$. In the present study nine variables, which were historically capable of explaining TMD in previous epidemiological studies $(2,5,6)$, were used and the final predictive model pointed out that pain, age and depression were the variables that could predict TMD in this sample.

Different studies have found association of low economic power and educational level with temporomandibular disturbance (5). However, in this model of prediction the socioeconomic variables were not present, and some reasons are suggested for this fact. The association of TMD with socioeconomic factors occurs because the lower social classes are more exposed to a greater load of stressor elements, such as less access to education, income, leisure, food and health services. These conditions may have repercussions on stress, anxiety and depression, which are also significantly related to TMD, and thus, make the isolated association of socioeconomic variables with TMD difficult. Therefore, as depression formed part of the predictive model, the socioeconomic variables could not predict TMD in an isolated manner.

In these results, age was the only demographic variable that remained in the predictive model. Higher prevalence of TMD was found as the age of individuals increased, with a peak prevalence occurring from 45 to 49 years of age $(41 \%)$ and decrease in the curve as from 60 years $(22.3 \%)$. In the predictive model, the two age-ranges from 25 to 44 years and 45 to 59 years were those that composed the Group with higher risk for TMD. The pattern of prevalence has been similar for population-based epidemiological surveys (19), and thus, the present study demonstrated that not only was age a recognized explanatory factor, but it was also a significant predictor of TMD. The pattern of age-related prevalence; that is, greater probability of TMD occurring in the age-range from 25 to 59 years, with a peak of prevalence between the 50s, and pattern of decline at 60 years, may be associated with biological and psychosocial reasons, or with factors related to the generation itself.

Due to the multifactorial nature of TMD and to the psychological factor being commonly studied as a variable that triggers this condition, some psychological disorder was expected to form part of the predictive model. For this model, the presence of depression, together with the other two variables - pain and age-range, enabled it to predict the probability of $55.1 \%$ of having TMD. These data corroborate the findings in the literature, because evaluation of the psychological profiles demonstrated that depression was one of the most common disorders in patients with TMD, and could be considered an important risk factor for triggering and maintaining the pathology (7).

The authors could perceive that various studies have corroborated this relationship, although, the most recent researches have demonstrated the need for more detailed explanations of the psychophysiology of stress to enable the association of TMD with depression to be understood $(20,21)$. Due to this presupposition, the importance of the hypothalamus/pituitary/adrenal axis (HPA), and the release of cortisol has been pointed out to explain the maintenance of psychological disorders in patients with TMD (22). Individuals with TMD exhibit characteristics of orofacial pain that may activate the hypothalamus/ pituitary/adrenal axis, which leads to an increase in serum 
cortisol release. Elevated levels of cortisol in patients with TMD may possibly be the physiological response to chronic pain and stress (23).

Individuals exposed to psychological stress have an increased risk for the occurrence and progression of TMD (22). The most frequent explanation suggests that when exposed to psychological problems, patients respond with abnormal movements of the mandible, parafunctional habits, facial pain and muscle hyperactivity. Excessive muscle force may cause biomechanical changes in the temporomandibular joint (TMJ), resulting in inflammation of the joint and retrodiscal tissue, in addition to articular pains $(10,22)$. This set of changes may increase the predisposition to the onset of TMD. Wu et al. (24), in their researches with rats, were able to demonstrate the relations between psychological disorder and change in the structures related to the TMJ, as well as change in the metabolism of the masticatory muscles, resulting in the appearance of symptoms in the TMJ.

Orofacial pain is the main cause of any association between TMD and psychosocial symptoms (10), and although it may be only one of the various symptoms of TMD verified, it is the one that most interferes in the $\vec{s}$ patients day-to-day life. The results of the present study demonstrated the dimension of this variable, considering that in spite of the demographic and psychosocial variables having formed part of the predictive model, the variable with the highest power of prediction was the one of a clinical nature - the presence of orofacial pain. It was the first, statistically significant variable capable of partitioning the sample into more homogeneous sub-groups, resulting in a probability of $47 \%$. The other possibilities resulting from age (51.9\%) and depression (55.1\%) depended on the presence of pain to predict TMD in this sample.

The predictive analysis resulting from this crosssectional study is coherent with the case-control, cohort, and systematic review researches that have recognized pain as one of the main risk factors for TMD. Two multivariate method were used by the Oppera (25) study with the purpose of identifying the predictors of TMD. The authors affirmed that the conditions of pre-existent pain are one of the most important risk factors for the onset of the dysfunction.

In addition to this study pointing out advantages such as the use of RDC/TMD, and the sampling process having been representative for the entire base population (users of SUS), this study also made contributions relative to the use of the classification tree for constructing the predictive model. Traditionally, researches in health have used logistic regressions for this purpose, however, they determined the mean effect of an independent variable for a dependent variable. Thus, when decisions are made based on the results of regression, they are directed towards the mean of the population, without taking into consideration the special needs of population sub-groups. Moreover, the classification tree conciliates clinical and statistical relevance of the set of data, resulting in a statistically rich model when compared with solely clinical or statistical approaches. The method is a bridge between statistical and scientific strictness and the ease of interpretations.

Limitations must also be considered, such as the single form of recruiting patients, which prevents generalization of the results to other cultural and racial realities. Moreover, as this was a cross-sectional study, no relationship of causality could be inferred from these results. There are also limitations of the decision tree method that users must be aware of. The classification tree should not be used on small samples. A continuous variable must be categorized before be analyzed by the classification tree. Once completed, the classification tree can only be applied in the area where it was built.

Nevertheless, the research cooperated with the advancement of knowledge about TMD for three main reasons. Firstly, by pointing out pain in the face as the best clinical predictor, a significant step was taken towards prevention of the dysfunction. At present, preventive dental care of this disease is practically non-existent, and when present, is performed without an adequate evaluation of risk. Secondly, this research paid greater attention to this dysfunction in this studied population, which lacks a larger volume of attendance, and requires better reception, right from the time of anamnesis. Thirdly, because it constructed a predictive model that could be applied as a tool in the early diagnosis of TMD.

The authors were able to conclude that for this sample, the group at high risk for the development of TMD was composed of clinical, psychosocial and demographic variables, namely orofacial pain, depression and age. The best predictor for TMD was the clinical variable orofacial pain.

\section{Resumo}

0 objetivo deste estudo foi construir um modelo preditivo que utiliza a análise estatística de árvore de classificação para predizer a ocorrência de disfunção temporomandibular, dividindo a amostra em grupos de alto e baixo risco para o desenvolvimento da doença. A utilização de abordagens estatísticas preditivas que facilitem o processo de reconhecimento e / ou previsão da ocorrência de disfunção temporomandibular é de interesse da comunidade científica, com o objetivo de fornecer aos pacientes soluções mais adequadas a cada caso. Trata-se de um estudo transversal analítico de base populacional que envolveu uma amostra de 776 individuos que procuraram atendimento médico ou odontológico nas Unidades de Saúde da Familia de Recife, PE, Brasil. A amostra foi submetida à anamnese por meio do instrumento Research Diagnostic Criteria for Temporomandibular Disorders. Os dados foram inseridos no software Statistical Package for the Social Sciences 20.0 e analisados pelo teste Qui-quadrado de Pearson para análise bivariada e pelo método de árvore de classificação para 
análise multivariada. A desordem temporomandibular pode ser prevista pela presença da dor orofacial, idade e depressão. 0 grupo de alto risco foi composto por individuos com dor orofacial, entre 25 e 59 anos e que apresentavam depressão. 0 grupo de baixo risco foi composto por individuos sem dor orofacial. Os autores puderam concluir que o melhor preditor para a disfunção temporomandibular foi a dor orofacial e que 0 modelo preditivo proposto pela árvore de classificação pode ser aplicado como ferramenta para simplificar a tomada de decisão em relação à ocorrência de disfunção temporomandibular.

\section{References}

1. Di Paolo C, D'Urso A, Papi P, Di Sabato F, Rosella D, Pompa G, et al. Temporomandibular Disorders and Headache: A Retrospective Analysis of 1198 Patients. Pain Res Manag 2017;2017:3203027.

2. Bagis B, Ayaz EA, Turgut $S$, Durkan R, Özcan M. Gender difference in prevalence of signs and symptoms of temporomandibular joint disorders: a retrospective study on 243 consecutive patients. Int J Med Sci 2012;9:539-544.

3. Vilanova LS, Gonçalves TM, Meirelles L, Garcia RC. Hormonal fluctuations intensify temporomandibular disorder pain without impairing masticatory function. Int J Prosthodont 2015;28:72-4.

4. Guarda-Nardini L, Piccotti F, Mogno G, Favero L, Manfredini D. Agerelated differences in temporomandibular disorder diagnoses. Cranio 2012;30:103-109.

5. Magalhães BG, de-Sousa ST, de Mello W, da-Silva-Barbosa AC, deAssis-Morais MP, Barbosa-Vasconcelos MM, et al. Risk factors for temporomandibular disorder: binary logistic regression analysis. Med Oral Patol Oral Cir Bucal 2014;19:e232-e236.

6. Bair E, Ohrbach R, Fillingim RB, Greenspan JD, Dubner R, Diatchenko L, et al. Multivariable modeling of phenotypic risk factors for first-onset TMD: the OPPERA prospective cohort study. J Pain 2013;14:T102-T115.

7. Tournavitis A, Tortopidis D, Fountoulakis K, Menexes G, Koidis P. Psychopathologic profiles of TMD patients with different pain locations. Int J Prosthodont 2017;30:251-257.

8. Kim TY, Shin JS, Lee J, Lee YJ, Kim MR, Ahn YJ, et al. Gender difference in associations between chronic temporomandibular disorders and general quality of life in Koreans: A Cross-Sectional Study. PLoS One 2015;10:e0145002.

9. Pelkonen ES, Mäki PH, Kyllönen MA, Miettunen JA, Taanila AM, Sipilä KK. Pain-related symptoms of temporomandibular disorders in the offspring of antenatally depressed mothers and depressed parents: a 31-year follow-up of the Northern Finland Birth Cohort 1966. Eur J Pain 2013;17:1048-1057.

10. Dougall AL, Jimenez CA, Haggard RA, Stowell AW, Riggs RR, Gatchel RJ. Biopsychosocial factors associated with the subcategories of acute temporomandibular joint disorders. J Orofac Pain 2012;26:7-16.

11. Chen $X$, Wang $M$, Zhang $H$. The use of classification trees for bioinformatics. Wiley Interdiscip Rev Data Min Knowl Discov
2011;1:55-63.

12. Li CP, Zhi XY, Ma J, Cui Z, Zhu ZL, Zhang $C$, et al. Performance comparison between Logistic regression, decision trees, and multilayer perceptron in predicting peripheral neuropathy in type 2 diabetes mellitus. Chin Med J (Engl) 2012;125:851-857.

13. Borges CM, Campos ACV, Vargas AMD, Ferreira EFe. Profile of tooth loss in adults according to social capital, demographic and socioeconomic characteristics. Cien Saude Colet 2014;19:1849-1858.

14. Piper ME, Loh WY, Smith SS, Japuntich SJ, Baker TB. Using decision tree analysis to identify risk factors for relapse to smoking. Subst Use Misuse 2011;46:492-510.

15. Ito $A$, Hayashi M, Hamasaki T, Ebisu S. Risk assessment of dental caries by using Classification and Regression Trees. J Dent 2011;39:457-463.

16. Morgan J. Classification and regression tree analysis 2014. Available from: https://www.bu.edu/sph/files/2014/05/MorganCART.pdf.

17. Dworkin SF, LeResche L. Research diagnostic criteria for temporomandibular disorders: review, criteria, examinations and specifications, critique. J Craniomandib Disord 1992;6:301-355.

18. Organization WH. Oral health surveys: basic methods. iris - Insitutional Repository for Information Sharing 1997:4:79.

19. Yekkalam N, Wänman A. Prevalence of signs and symptoms indicative of temporomandibular disorders and headaches in 35-, 50-, 65- and 75-year-olds living in Västerbotten, Sweden. Acta Odontol Scand 2014;72:458-465.

20. Muzalev K, Visscher CM, Koutris M, Lobbezoo F. Long-term variability of sleep bruxism and psychological stress in patients with jawmuscle pain: Report of two longitudinal clinical cases. J Oral Rehabil 2018;45:104-109.

21. Ohrbach R, Michelotti A. The role of stress in the etiology of oral parafunction and myofascial pain. Oral Maxillofac Surg Clin North Am 2018;30:369-379.

22. Almeida Cd, Paludo A, Stechman-Neto J, Amenábar JM. Saliva cortisol levels and depression in individuals with temporomandibular disorder: preliminary study. Rev Dor 2014;15:169-172.

23. Nadendla LK, Meduri V, Paramkusam G, Pachava KR. Evaluation of salivary cortisol and anxiety levels in myofascial pain dysfunction syndrome. Korean J Pain 2014;27:30-34.

24. Wu G, Chen L, Zhu G, Su Y, Chen Y, Sun J, et al. Psychological stress induces alterations in temporomandibular joint ultrastructure in a rat model of temporomandibular disorder. Oral Surg Oral Med Oral Pathol Oral Radiol Endod 2011;112:e106-e112.

25. Sanders AE, Slade GD, Bair E, Fillingim RB, Knott $C$, Dubner $R$, et al. General health status and incidence of first-onset temporomandibular disorder: the OPPERA prospective cohort study. J Pain 2013;14:T51-T62.

Received September 26, 2019 Accepted February 17, 2020 